\title{
The effect of retrieval enactment on recall of subject-performed tasks and verbal tasks
}

\author{
REZA KORMI-NOURI, LARS NYBERG, and LARS-GÖRAN NILSSON \\ University of Umeå, Umeå, Sweden
}

\begin{abstract}
The effect of retrieval enactment on memory for nouns (objects) or verbal phrases describing simple actions (e.g., "lift the box") was addressed in two experiments. In Experiment 1, the type of object involved in the actions was manipulated, with three different types of object being used (body parts, laboratory-related objects, and external objects). In Experiment 2, the integration between the verb-noun pairs was manipulated (well-integrated vs. poorly integrated). Results from both experiments showed that whereas encoding enactment (motor encoding and verbal test) substantially improved the memory performance compared with a verbal condition (verbal encoding and verbal test), retrieval enactment (verbal encoding and motor test) had no major impact on the number of recalled nouns or phrases. Moreover, there was no additional effect of dual enactment (motor encoding and motor test). The overall pattern of the results suggests that there is a fundamental difference between motor processing at encoding and motor processing at retrieval, and the lack of encoding specificity advantage for the motor modality contradicts the view that encoding enactment of verbal commands results in storage of motor representations.
\end{abstract}

The subject-performed task (SPT) was developed a decade ago by Cohen (1981) and, independently, by Engelkamp and Krumnacker (1980), as a new experimental paradigm to study memory. The general procedure is that subjects are presented with verbal commands (e.g., "roll the ball," "break the match") and are asked to perform the action indicated by each command. In a subsequent test, the subjects are instructed to recall the commands presented. In the typical control condition [the verbal task (VT)], the subjects are presented with the same verbal commands as in the SPT condition, but without any instruction to perform the actions. The standard result in such experiments is that recall of commands after enactment is higher than recall of commands without any enactment. This SPT effect has been obtained in a wide variety of experimental settings (see Cohen, 1989 , for a review).

The main body of research in this area has thus explored the role of enactment during encoding, whereas, to our knowledge, the role of enactment at retrieval has been examined in only four studies.

\section{PREVIOUS STUDIES OF RETRIEVAL ENACTMENT}

In a study by Saltz and Dixon (1982), subjects were given 12 simple action sentences (e.g., "the workman was digging a hole in the ground," "the doctor fell asleep

This research was supported by a grant to L.-G.N. from the Swedish Council for Research in the Humanities and Social Sciences. Correspondence concerning this article should be sent to R. Kormi-Nouri, Department of Psychology, University of Umeå, S-901 87 Umeå, Sweden. in the chair") to learn in two conditions-enactment and nonenactment. In the enactment condition, the subjects were told to act out each sentence after they had repeated the sentence, which was first read by the experimenter. In the nonenactment condition, the subjects were instructed to repeat the sentence twice without any enactment. The subjects were later tested by cuing with the verb phrase of each sentence. During recall, they either were or were not instructed to enact the verb cue before attempting to recall each sentence. The result showed that motoric enactment at encoding doubled recall performance, irrespective of whether enactment of the cues was attempted during the recall phase. Motoric enactment at retrieval only (i.e., in the absence of encoding enactment) did not improve recall of the sentences. In a subsequent study, Saltz (1988) replicated this finding of no effect of retrieval enactment on recall performance.

In a more recent study by Norris and West (1993), subjects were presented with 32 actions involving body parts (e.g., "snap your fingers," "cross your legs"). There were two different encoding conditions - enactment and nonenactment. At retrieval, a free recall test was given to the subjects in two different conditionsagain, enactment and nonenactment. An SPT effect was obtained when enactment occured either at encoding or at retrieval. Norris and West (1993) therefore concluded that the presence of the motor modality at either stage of processing enhances recall. However, dual enactment (i.e., the combination of motor encoding and motor retrieval) did not have an additive effect compared with either single encoding enactment or single retrieval enactment.

In another recent study (Brooks \& Gardiner, 1994), subjects were presented with 24 action phrases (e.g., "fly 
a kite," "beat an egg") which involved imaginary objects in three conditions: (1) verbal encoding and verbal retrieval (verbal control); (2) SPT encoding and verbal retrieval (retrospective SPTs); and (3) verbal encoding and SPT retrieval (prospective SPTs). Brooks and Gardiner found that, compared with the verbal controls, the subjects benefited from the SPTs at encoding (retrospective SPTs) but not from the SPTs at retrieval (prospective SPTs).

\section{THE PRESENT STUDY}

The studies by Saltz (1988), Saltz and Dixon (1982), and Norris and West (1993) thus tested both dual enactment [motor encoding and motor test (MM-condition)] and single retrieval enactment [verbal encoding and motor test (VM-condition)], whereas the study by Brooks and Gardiner (1994) examined only the VM condition. Interestingly, neither the study by Saltz and Dixon nor the one by Norris and West found an increased effect of dual enactment compared with regular encoding enactment. This finding is somewhat surprising because, on the basis of the encoding specificity principle (Tulving \& Thomson, 1973), it can be predicted that enactment at both study and test should result in a better recall performance than a condition with enactment only at one of these stages. Therefore, the first purpose of the present study was to test the replicability of this finding. To do so, the items we used were the standard verb-noun pairs rather than the type of action sentences used by Saltz and Dixon (1982). We included three different types of item in the experiment. In addition to items involving objects related to the body (i.e., body parts), as in Norris and West's (1993) study, we used items related to the laboratory (e.g., "tap the table") and those involving external objects (e.g., "lift the box").

For the VM condition, the findings are inconsistent. Saltz (1988; Saltz \& Dixon 1982) and Brooks and Gardiner (1994) found no effect of retrieval enactment in the absence of encoding enactment, whereas Norris and West (1993) did find such an effect. A possible explanation for these discrepant findings concerns the types of verb-noun items used. As noted above, Norris and West used only those involving body parts, whereas Saltz and Dixon and Brooks and Gardiner used items involving imaginary external objects. It is possible that an effect of retrieval enactment occurs for items that involve objects that are present at retrieval (e.g., body parts and objects related to the laboratory), but not for items that involve objects that are absent (external objects). Another possibility is that there is something special about items involving body parts that restricts the effect of retrieval enactment to this type of item. The inclusion of two different types of items involving objects that are present at retrieval — body parts and laboratory objectsas well as of items involving objects that are absent at retrieval allowed us to test these different possibilities.

To summarize, two main issues were addressed in the present study: (1) the impact of dual (i.e., encoding and retrieval) enactment of action verbs on recall performance; and (2) the impact of single retrieval enactment on recall performance. To test the generalizability of previous findings of no effect of dual enactment and an effect of retrieval enactment only for items involving body parts, three different types of object were used.

\section{EXPERIMENT 1}

\begin{abstract}
Method
Design. The design was a 2 [type of encoding (SPT vs. VT)] $\times 2$ [retrieval condition (verbal vs. enacted)] $\times 3$ [type of item (involving body parts, laboratory-related objects, or external objects)] mixed design. Retrieval condition was a between-subjects factor and type of encoding condition and type of item were withinsubjects factors.
\end{abstract}

Materials. Thirty commands in two different orders were used as the to-be-remembered materials. Half of the subjects were given the first order, and the other half the second order. Half of the items were presented as SPTs and the other half as VTs. The nouns in the commands were from three different categories (10 nouns per category): (1) body parts (e.g., "shake the head"); (2) laboratoryrelated objects (e.g., "look at the door"); and (3) external objects from outside the laboratory (e.g., "roll the marble"). In the CR test, the verbs were given as cues.

Subjects. Twenty-four naive subjects from the city of Umeå were randomly assigned to the two between-subjects conditions, verbal test and enacted test (with 12 subjects in each condition). The age ranges in the different conditions were $17-37$ years $(M=$ 23.33) for the verbal-test condition and 16-30 years $(M=20.83)$ for the enacted-test condition. The subjects were paid the equivalent of $\$ 7$ each for participating in the experiment, which lasted about $25 \mathrm{~min}$

Procedure. The commands were written on cards which were shown one at a time by the experimenter at a rate of $6 \mathrm{sec}$ per item. The interstimulus interval was $3 \mathrm{sec}$. The subjects were instructed to try to remember the noun of each command. For VTs, the subjects were instructed only to read the sentences aloud, while for SPTs, they were instructed to read the sentences aloud and then to perform the actions indicated by the commands. For SPTs involving external objects, the objects were given to the subjects at the time of presentation of the commands, and were then hidden after performing the actions. For SPTs involving body parts and laboratory-related objects, the experimenter pointed to the objects. The experimenter said "action" before presenting SPTs and "sentence" before presenting VTs. All of the subjects were given an example of the VTs and an example of the SPTs before the study list was presented. Moreover, before presentation of the study list, the subjects were informed about an unspecified subsequent memory test.

After the presentation of the study list, the subjects were given a 30-item vocabulary test. The main purpose of this test was to eliminate any effects of short-term memory, but another reason for including it was to assess subjects' word comprehension in the two different conditions. Mean performance was $24.08(S D=2.64)$ for the verbal-test condition and $24.25(S D=4.58)$ for the enactedtest condition. There was no significant difference between the two conditions with respect to verbal ability $(p>.90)$.

At recall, subjects assigned to the verbal-test condition were given a standard CR test. That is, the cues were printed on cards and the subjects were shown them one at a time (each cue was shown for $10 \mathrm{sec}$ ) and were asked to write down the corresponding nouns on a sheet of paper.

Subjects assigned to the enacted-test condition received the same instructions as subjects in the verbal-test condition. In addition, they were instructed to enact the action verbs at retrieval. That is, the subjects were asked to perform the actions indicated by the cues, and following enactment, they were instructed to try 
to write down the nouns that were presented with the verbs at study (i.e., the objects with which they performed the tasks at study).

\section{Results and Discussion}

A strict procedure of scoring recall of the nouns was used, whereby responses were accepted only if they were exactly the same as those presented in the study list. The results are shown in Table 1.

As can be seen from Table 1, there was a strong effect of encoding enactment. There was no overall effect of enactment at retrieval for SPTs, whereas VTs were affected by retrieval enactment. Note, though, that for VTs, the effect of retrieval enactment was primarily restricted to items involving body parts. Finally, it can be seen that encoding enactment led to a markedly higher recall performance than did retrieval enactment $(.71$ vs. $.31)$.

A 2 [retrieval condition (verbal vs. enacted)] $\times 2$ [encoding condition (SPT vs. VT)] $\times 3$ [type of item (involving body parts, laboratory-related objects, or external objects)] mixed analysis of variance (ANOVA) was performed to statistically evaluate the effect of encoding and retrieval enactment across the different types of item used. There was a significant effect of encoding enactment [i.e., an SPT effect; $F(1,22)=143.8, M S_{\mathrm{e}}=1.24$, $p<.001$ ], but no effect of retrieval enactment $(p>.20)$. There was also a main effect of type of item $[F(2,44)=$ $\left.8.67, M S_{\mathrm{e}}=.95, p<.001\right]$, and post hoc analyses revealed that items involving body parts were significantly better recalled than those involving laboratory-related objects or external objects $(p<.01)$ and that there was no difference in terms of recall between the two latter types of item.

The interaction between the within-subjects factors was significant $\left[F(2,44)=3.5, M S_{\mathrm{e}}=1.10, p<.05\right]$, reflecting that for VTs, but not for SPTs, items involving body parts were better recalled than those involving external objects.

The interaction between encoding enactment and retrieval enactment was marginally significant $[F(1,22)=$ $\left.3.80, M S_{\mathrm{e}}=1.24, p=.06\right]$, indicating that whereas retrieval enactment did not have any effect on SPTs, there was a tendency toward an effect for VTs. As can be seen from Table 1, this tendency was mainly caused by an increase in performance for the items involving body parts (see Norris \& West, 1993), and the interaction disappeared $(p>.70)$ when the same analysis was conducted

Table 1

Mean Proportions of Correctly Recalled Nouns as a Function of Encoding Condition, Retrieval Condition, and Type of Object in Experiment 1

\begin{tabular}{cccccccc}
\hline & \multicolumn{4}{c}{ Retrieval Condition } \\
\cline { 2 - 5 } \cline { 5 - 8 } Encoding Condition & BP & EX & LB & & BP & EX & LB \\
\hline SPT & .80 & .76 & .56 & & .70 & .70 & .66 \\
VT & .26 & .14 & .18 & .52 & .16 & .26 \\
\hline
\end{tabular}

Note-BP = body parts; EX = external objects; LB = laboratory-related objects. after exclusion of those items. In addition, a 2 [test (verbal vs. enacted)] $\times 2$ [item (body parts vs. non-body parts)] simple effects analysis on the VT data revealed that whereas there was an effect of retrieval enactment for items involving body parts $(p<.01)$, there was no such effect for those not involving body parts $(p>.80)$.

In sum, the main findings of Experiment 1 were: (1) an effect of encoding enactment but no additional effect of dual enactment; and (2) an effect of retrieval enactment that was restricted to items involving body parts. The main purpose of Experiment 2 was to try to shed some light on the latter finding; its secondary purpose was to replicate the first finding.

\section{EXPERIMENT 2}

The fact that the effect of retrieval enactment is present for items involving body parts but not for those involving external objects is puzzling. One possible explanation for this diverse pattern of results is that enactment at retrieval is helpful for memory performance when enacting improves cue effectiveness, and that such an improvement is particularly likely to occur for items involving body parts. This may be due to the relation between action verbs and body parts. Experiment 1 showed that body parts are of importance in action events. In addition, for normative data collected by Kormi-Nouri (1994), it was found that some action verbs (e.g., stretch, compress, shake, wiggle) are strongly integrated with body parts. Thus, it is possible that actions involving body parts involve a high integration between action verbs and objects, and the action verbs therefore direct the subjects to the correct noun. According to such an explanation, the fact that the effect of retrieval enactment is restricted to body parts might be a consequence of cue-target integration.

To test this hypothesis, in Experiment 2, only items involving external objects were used. Some of these were well-integrated, while others were poorly integrated. In well-integrated items, there is a logical or functional relation between action verb and object (e.g., "write with the pen," "measure with the ruler"), whereas there is no such relation in poorly integrated items (e.g., "lift the glass," "look at the candle"). The normative data (KormiNouri, 1994) have shown that the number of possible objects generated when the action verbs are given without prior study is significantly higher for poorly integrated items than it is for well-integrated items. If the effect of retrieval enactment for items involving body parts is due to a strong cue-target relationship for these items, there should be a stronger effect of retrieval enactment for well-integrated items than for poorly integrated items.

The procedure in Experiment 2 differed in two respects from that used in Experiment 1. First, all subjects were instructed to remember "whole sentences" or "whole actions" (i.e., not just nouns). This modification was made because the test used was CR, and hence the instruction used in Experiment 1 only to encode nouns (objects) might have hindered the formation of associa- 
tions between verbs and nouns (which in turn should make the cues less effective). Second, to facilitate enactment of the action verbs during retrieval, the subjects were given a piece of wood (a neutral object) with which to perform the actions. This procedural change was made because while running Experiment 1, it was noted that some action verbs might not be properly performed without any object.

\section{Method}

Design. The design was a 2 [type of encoding (SPT vs. VT)] $\times$ 2 [type of retrieval (verbal vs. enacted)] $\times 2$ [type of item (wellintegrated vs. poorly integrated)] mixed design, with type of retrieval varying between subjects and type of encoding and type of item varying within subjects.

Subjects. Twenty-four naive subjects from the city of Umea were randomly assigned to the two retrieval conditions, verbal test and enacted test (with 12 subjects in each condition). Age ranges were $17-33$ years $(M=24.25)$ for the verbal-test condition, and $17-31$ years $(M=24.17)$ for the enacted-test condition. The conditions did not differ with respect to verbal ability of the subjects $(p>.40)$. Mean performance, for a 30 -item vocabulary test, was $24.67(S D=1.44)$ for the verbal-test condition and $25.42(S D=$ 3.09) for the enacted-test condition. The subjects were paid the equivalent of $\$ 7$ each for their participation. The experiment lasted approximately $25 \mathrm{~min}$.

Materials. A new study list of 28 items (e.g., "write with the pen," "lift the glass"), presented in two different orders, was used. It included 14 VTs and 14 SPTs. The experimenter said "sentence" before presenting the VTs and "action" before presenting the SPTs. Half of the items (7 VTs and 7 SPTs) were well-integrated, while the other half were poorly integrated.

Procedure. Except for the above-noted modifications, the procedure was identical to that used in Experiment 1.

\section{Results and Discussion}

As in Experiment 1, a strict scoring of noun (object) recall was adopted. The results are shown in Table 2.

As can be seen from Table 2, there was an effect of encoding enactment but no effect of dual enactment. In fact, the performance following single encoding enactment was higher than that following dual enactment. Moreover, in contrast to Experiment 1, there was no tendency toward an effect of retrieval enactment for VTs, but instead there was a higher performance for the VV condition than for the VM condition. It should also be noted that the decrease in performance from the nonenactment retrieval conditions to the enactment retrieval conditions was obtained for both types of items, and was even more pronounced for the poorly integrated items.

Table 2

Mean Proportions of Correctly Recalled Nouns as a Function of Encoding Condition, Retrieval Condition, and Type of Object in Experiment 2

\begin{tabular}{ccccc}
\hline & \multicolumn{3}{c}{ Retrieval Condition } \\
\cline { 2 - 4 } Encoding Condition & \multicolumn{2}{c}{ Verbal } & & \multicolumn{2}{c}{ Enacted } \\
\cline { 2 - 5 } & WI & PI & WI & PI \\
\hline SPT & .93 & .60 & .87 & .49 \\
VT & .73 & .23 & .69 & .11 \\
\hline
\end{tabular}

Note-WI = well-integrated items; $\mathrm{PI}=$ poorly integrated items.
A 2 [retrieval condition (verbal vs. enacted)] $\times 2$ [encoding condition (SPT vs. VT)] $\times 2$ [type of item (wellintegrated vs. poorly integrated)] mixed ANOVA supported these general impressions by showing a significant effect of encoding enactment $[F(1,22)=49.6$, $\left.M S_{\mathrm{e}}=1.9, p<.001\right]$, but no effect of retrieval enactment $(p>.10)$.

There was also a main effect of type of item $[F(1,22)=$ $\left.180.3, M S_{\mathrm{e}}=1.3, p<.001\right]$, showing that well-integrated items were recalled to a higher extent than were poorly integrated items. The interaction between encoding condition and type of item was also significant $[F(1,22)=$ $\left.5.05, M S_{\mathrm{e}}=1.98, p<.05\right]$, reflecting a stronger effect of encoding enactment for poorly integrated items than for well-integrated items. This suggests that encoding enactment may increase the integration between action verbs and objects. Neither the interaction between type of test and type of item nor the three-way interaction between type of encoding, type of test, and type of item were significant $(p s>.30)$.

As a whole, the results of Experiment 2 replicated the findings in Experiment 1 of an effect of encoding enactment and an absence of an effect of both retrieval enactment and dual enactment. The lack of effect of single retrieval enactment $(V M<V V)$ suggests that the effect of single retrieval enactment found both in Experiment 1 and by Norris and West (1993) was due to the presence of objects at retrieval. That is, retrieval enactment will be effective if the objects are present at the time of test.

\section{GENERAL DISCUSSION}

The present study has shown that, in general, retrieval enactment does not improve memory for SPTs and VTs. The lack of effect of retrieval enactment on memory performance is in line with previous findings by Saltz (1988; Saltz \& Dixon, 1982) and Brooks and Gardiner (1994), but not with those by Norris and West (1993), who recently demonstrated a reliable SPT retrieval effect. ${ }^{1}$

Norris and West (1993), however, only used items related to the body. A proper comparison of the present data and their results should therefore focus on the figures in Table 1 for items involving body parts, in which case the results of the two studies are in perfect agreement: The performance is highest for motor encoding and verbal test (MV), followed by motor encoding and motor test (MM), verbal encoding and motor test (VM), and verbal encoding and verbal test (VV).

Thus, both in the present study and in Norris and West's (1993) study, the performance is actually higher for motor encoding followed by a verbal test than it is for motor encoding followed by a test involving enactment. To explain why the dual modality processing (the combination of motor and verbal processing during encoding and retrieval) results in a recall level that is comparable to (or higher than) single modality processing (motor encoding and motor retrieval), Norris and West suggested a distinctiveness explanation; they proposed 
that the lack of support for the encoding specificity principle for the single modality condition was due to the fact that motor processing at either stage (encoding or retrieval) is so distinctive that additional motor processing is of limited value.

The present data, however, cause us to raise some serious objections to this explanation. ${ }^{2}$ First, in our data, there was a considerable increase in recall performance between the VM and $\mathrm{MM}$ conditions, suggesting that enactment at encoding is of special importance for good memory performance. Moreover, the effect of retrieval enactment seems to be restricted to those that involve items that do not involve extraenvironmental objects (and particularly to body parts). In Experiment 2, in which only items involving external objects were used, the VV condition actually exceeded the VM condition, thereby providing further support for the view that encoding enactment holds a particular status and that retrieval enactment is not very helpful for memory performance.

Thus, the outcome of the present experiments clearly indicates that whereas encoding enactment substantially improves memory performance, retrieval enactment is of limited importance. As a result of this outcome, which casts doubt on the distinctiveness explanation put forward by Norris and West (1993), a somewhat different explanation is put forward here-namely, that there is a lack of encoding specificity advantage for the MM condition over the MV condition because motor cues are not effective. We believe that this is because what has been stored is in a verbal rather than a motor code (see Helstrup, 1986; Nyberg, 1993). To the extent that retrieval enactment is effective (as it was for items involving body parts), this is most likely because enactment increases the efficiency of the cue, not because the storage and retrieval formats match.

This view - that the lack of support for the encoding specificity principle is due to the fact that the to-beremembered information is stored in a verbal code-is clearly at odds with the view that encoding enactment results in the storage of some sort of motor representation, or motor program (e.g., Engelkamp, 1990; Engelkamp \& Zimmer, 1984, 1985). If the encoded information had been in some sort of motoric code, motor cues would surely have been effective, and an encoding specificity advantage would have been found.

Turning now to the effect of single retrieval enactment, the general conclusion to be drawn from the present experiments is that retrieval enactment has no major effect on memory performance. In light of the strong effect of encoding enactment on memory performance, this lack of effect of retrieval enactment suggests a fundamental difference between motor processing at encoding and that at retrieval. The core of this difference is, in our mind, that whereas the role of encoding enactment is to improve the encoding, the role of retrieval enactment is to increase the accessibility of the information to be retrieved. In short, encoding enactment is a form of encoding support, while retrieval enactment is a form of retrieval support; as for retrieval support in general (e.g., Tulving \& Thomson, 1973), the extent to which a given retrieval support is effective depends on its match with the informational content of the memory trace.

The view put forward here- that motor processing at encoding is fundamentally different from that at retrieval - is in close agreement with the findings and conclusions by Brooks and Gardiner (1994). One of the most striking and well-researched findings within the domain of SPT memory is that encoding enactment either eliminates age effects in free recall (e.g., Bäckman $\&$ Nilsson, 1984, 1985) or greatly reduces them (e.g., Nyberg, Nilsson \& Bäckman, 1992). For retrieval enactment, however, the picture appears to be quite different. Brooks and Gardiner demonstrated reliable age effects also in the presence of eliminated age effects following encoding enactment. Thus, the facilitation of encoding enactment for older adults is apparently not matched by retrieval enactment. Note, though, that in one of the conditions reported in the Norris and West (1993) study, motor processing at retrieval improved the performance of the older subjects, but not that of the younger subjects, to the extent that a nonsignificant age effect emerged. Since Brooks and Gardiner used items involving (imaginary) external objects and not objects related to the body, the difference in results once again concerns the type of items used. This matter will be considered next.

The apparent effect of retrieval enactment following verbal encoding of items not involving external objects, as demonstrated in both Experiment 1 and by Norris and West (1993), is striking. We refute a distinctiveness explanation of this effect and suggest instead an alternative explanation. Retrieval enactment may be helpful for memory performance in cases in which enacting improves cue effectiveness, and it is plausible that such an improvement only occurs for items involving objects that are present at retrieval. This is because for these items (and for items involving body parts in particular), the enactment cues can be integrated with the objects. For example, if the cue is "shake" and the target is "the head," and the subject is instructed to enact the cue, it is likely that the subject will shake his or her head and thereby be reminded that the correct target is "the head". In such cases, it is therefore reasonable to assume that retrieval enactment increases the likelihood that the cue will lead the subject to the correct target. If, instead, the cue is "lift" and the target is "the box," enactment (in the absence of the object) should not improve cue effectiveness and may even reduce it. This line of reasoning is in agreement with the findings in Experiment 2 of no effect of retrieval enactment for well-integrated items and of a negative effect of retrieval enactment for poorly integrated items. The external objects were not available at retrieval, whereas body parts were. Furthermore, the results of Experiment 2 showed that encoding enactment increases the integration between action verbs and objects, but that retrieval enactment does not. The lack of effect of retrieval enactment may also be due to the ab- 
sence of objects at test. Clearly, this explanation should be put under experimental scrutiny, but it is, nevertheless, consistent with a substantial amount of empirical observation.

In conclusion, the major purpose of the present study was to explore the effect of retrieval enactment on recall of verbal commands following encoding enactment and verbal encoding. The finding that retrieval enactment has no major impact on memory performance indicates a fundamental difference between motor processing at encoding and motor processing at retrieval, and the lack of encoding specificity advantage for the motor modality contradicts the view that encoding enactment of verbal commands results in storage of motor representations of the to-be-remembered material.

\section{REFERENCES}

BÄCKMAN, L., \& Nilsson, L.-G. (1984). Aging effects in free recall An exception to the rule. Human Learning, 3, 53-69.

Bäckman, L., \& NiLsson, L.-G. (1985). Prerequisites for lack of age differences in memory performance. Experimental Aging Research 11, 67-73.

Brooks, B. M., \& Gardiner, J. M. (1994). Age differences in memory for prospective compared with retrospective subject-performed tasks. Memory \& Cognition, 22, 27-33

COHEN, R. L. (1981). On the generality of some memory laws. Scandinavian Journal of Psychology, 22, 267-282.

CoHEN, R. L. (1989). Memory for action events: The power of enactment. Educational Psychology Review, 1, 57-80.

ENGELKAMP, J. (1990). Memory of action events and some implications for imagery. In C. Cornoldi \& McDaniel (Eds.), Imagery and cognition (pp. 183-219). New York: Springer-Verlag.

ENGELKAMP, J., \& KRUMNACKER, H. (1980). Imaginale und motorische Prozesse beim Behalten verbalen Materials. Zeitschrift für experimentelle und angewandte Psychologie, 27, 511-533.

ENGElKaMP, J., \& ZiMmeR, H. D. (1984). Motor program information as a separable memory unit. Psychological Research, 46, 283-299.

ENGELKAMP, J., \& ZiMmeR, H. D. (1985). Motor programs and their relation to semantic memory. German Journal of Psychology, 9, 239-254.
Helstrup, T. (1986). Separate memory laws for recall of performed acts? Scandinavian Journal of Psychology, 27, 1-29.

KORMI-NOURI, R. (1994). The role of integration in memory for enacted and nonenacted events. (Manuscript submitted for publication.)

NORRIS, M. P., \& WEST, R. L. (1993). Activity memory and aging: The role of motor retrieval and strategic processing. Psychology \& Aging, 8, 81-86

NYBERG, L. (1993). The enactment effect: Studies of a memory phenomenon. Unpublished doctoral dissertation, University of Umeå, Sweden.

Nyberg, L., Nilsson, L.-G., \& Bäckman, L. (1992). Recall of actions, sentences and nouns: Influence of adult age and passage of time. Acta psychologica, 79, 1-10.

SALTZ, E. (1988). The role of motoric enactment (M-processing) in memory for words and sentences. In M. M. Gruneberg, P. E. Morris, \& R. N. Sykes (Eds.), Practical aspects of memory: Current research and issues (Vol. 1, pp. 408-414). Chichester, U.K.: Wiley. SALTZ, E., \& DrXon, D. (1982). Let's pretend: The role of motoric imagery in memory for sentences and words. Journal of Experimental Child Psychology, 34, 77-92.

Tulving, E., \& ThOMson, D. M. (1973). Encoding specificity and retrieval processes in episodic memory. Psychological Review, 80, 353-373.

\section{NOTES}

1. For younger adults, this effect was found for an unorganized list only, whereas older adults benefited from retrieval enactment following verbal encoding for both organized and unorganized lists.

2. It should be pointed out that we are in agreement with Norris and West (1993) that the superior memory performance following encoding enactment is to be explained in terms of distinctiveness. In fact, Nyberg (1993) proposed a distinctiveness model in which it was suggested that enactment at encoding increases the distinctiveness of the memory traces by improving item-specific as well as relational processing (the latter including both categorical integration and event integration). The major point of disagreement is that, in contrast to Norris and West, we do not believe that retrieval enactment "is so distinctive that additional processing [at encoding] is of no value for remembering the item" (1993, p. 85).

(Manuscript received October 21, 1993; revision accepted for publication March 18, 1994.) 